\title{
Static and dynamic properties of low-temperature order in the one-dimensional semiconductor $\left(\mathrm{NbSe}_{4}\right)_{3} \mathrm{I}$
}

\author{
D. Dominko* \\ Institute of Physics, Bijenička c. 46, 10000 Zagreb, Croatia \\ and Institute of Physics, Johannes Gutenberg-University, 55099 Mainz, Germany \\ S. Vdović, H. Skenderović, D. Starešinić, and K. Biljaković \\ Institute of Physics, Bijenička c. 46, 10000 Zagreb, Croatia \\ D. Ristić and M. Ivanda \\ Center of Excellence for Advanced Materials and Sensing Devices, Ruđer Bošković Institute, Bijenička c. 54, 10000 Zagreb, Croatia
}

\section{J. E. Lorenzo}

Institut Néel, CNRS-UJF, 38042 Grenoble, France

\author{
J. Demsar \\ Institute of Physics, Johannes Gutenberg-University, 55099 Mainz, Germany \\ (Received 11 August 2016; published 28 September 2016; corrected 21 October 2016)
}

\begin{abstract}
We investigated static and dynamic lattice properties in a quasi-one-dimensional charge-ordered semiconductor $\left(\mathrm{NbSe}_{4}\right)_{3} \mathrm{I}$ by using Raman, femtosecond pump-probe spectroscopy and x-ray diffraction. In addition to a welldocumented pseudo-Jahn-Teller ferrodistortive structural transition at $T_{C}=274 \mathrm{~K}$, where the displacements of $\mathrm{Nb}$ ions lead to ferroelectric (FE) in-chain polarization with opposite direction in adjacent chains, all methods suggest an additional lowering of symmetry at $T^{*} \approx 160 \mathrm{~K}$. Although antiferroelectric (AFE) phase is partially formed at $T_{C}$, our results consistently point to an enhancement of the interchain order at $T^{*}$, thus leading to AFE order-disorder transition, as supported by the earlier dielectric and structural studies.
\end{abstract}

DOI: 10.1103/PhysRevB.94.104113

\section{INTRODUCTION}

$\left(\mathrm{NbSe}_{4}\right)_{3} \mathrm{I}$ is the only representative of one-dimensional halogenated transition metal tetrachalcogenides of the $\left(\mathrm{MX}_{4}\right)_{n} \mathrm{Y}$ family $(\mathrm{M}=\mathrm{Nb}$, Ta; $\mathrm{X}=\mathrm{S}, \mathrm{Se} ; \mathrm{Y}=\mathrm{I}, \mathrm{Br})$ that does not undergo the Peierls transition into the charge density wave (CDW) ground state. Instead, a structural ferrodistortive transition without noticeable superstructure has been observed. $\left(\mathrm{NbSe}_{4}\right)_{3} \mathrm{I}$ has a chainlike crystal structure, with each chain consisting of $\mathrm{Se}_{4}^{4-}$ rectangles separated by $\mathrm{Nb}^{4+} / \mathrm{Nb}^{5+}$ ions, while $\mathrm{I}^{-}$ions form columns linking the chains, Fig. 1(a). A valence of $\mathrm{Nb}$ ions in a simplified ionic picture is assumed to be ordered in the $4+4+5+$ fashion [1]. Here $\mathrm{Nb}^{4+}-\mathrm{Nb}^{4+}$ separation $(S)$ was found to be shorter than $\mathrm{Nb}^{4+}-\mathrm{Nb}^{5+}$ separation $(L)$, resulting in $L L S L L S$ stacking along the chain, with $L=3.25 \AA$ and $S=3.06 \AA$. Due to the rotation of $\mathrm{Se}_{4}$ rectangles around the chain direction ( $c$ axis), the unit cell consists of two chains with six $\mathrm{Nb}$ ions per chain. At room temperature (RT) the space group is $P 4 / m n c\left(D_{4 h}^{6}\right)$.

At $T_{C}=274 \mathrm{~K}\left(\mathrm{NbSe}_{4}\right)_{3} \mathrm{I}$ undergoes a structural phase transition from space group $P 4 / m n c\left(D_{4 h}^{6}\right)$ to $P 42_{1} c\left(D_{2 d}^{4}\right)$ [2]. The detailed $\mathrm{x}$-ray diffraction (XRD) study [3] at $128 \mathrm{~K}$ suggested that each $\mathrm{Nb}^{5+}$ ion is slightly displaced in the $c$ direction, towards one of the neighboring $\mathrm{Nb}^{4+}$ ions. Thereby the inversion symmetry is broken. However, these displacements are in the opposite directions between the neighboring chains, resulting in an unusual ferrodistortive AFE

\footnotetext{
*ddominko@ifs.hr
}

phase, Fig. 1(b). The loss of inversion symmetry is actually twofold, as the chains are shifted with respect to each other as well. Since the two $L$ distances are no longer equal, new in-chain LISLIS stacking is formed, with $L=3.31 \AA$ and $I=3.17 \AA$ A. Accordingly, dielectric spectroscopy [4] reveals colossal dielectric response with maximum around $160 \mathrm{~K}$, where $\epsilon_{r} \approx 10^{5}$. Using the space group analysis, it was proposed [5] that this ferrodistortive transition is driven by mixing the valence and conduction bands by phonons of appropriate symmetry, i.e., by the pseudo-Jahn-Teller (pJT) mechanism. While the scenario normally requires a narrow band gap, it was argued recently that the pJT distortion can take place even if the gap is wider, provided that the electron-phonon interaction is strong enough [6].

Transport studies revealed semiconducting behavior above $T_{C}$ with an activation energy of $0.19 \mathrm{eV}$ [1]. The symmetry change at $T_{C}$ is accompanied by changes in electronic properties. Systematic transport studies on a series of samples revealed two types of behavior in $\left(\mathrm{NbSe}_{4}\right)_{3} \mathrm{I}$, despite the fact that the samples were indistinguishable according to the XRD at RT [1]. In the case of type-I compound the activation energy is reduced at the $T_{C}$ and the low activation energy $(\approx 20 \mathrm{meV})$ persists at least down to $80 \mathrm{~K}$. In the type-II samples the resistivity exhibits plateau below $T_{C}$ and down to $T^{*} \approx 160 \mathrm{~K}$, below which the activation regime is restored with the activation energy of $\approx 0.13 \mathrm{eV}$. Our samples display similar behavior, as shown in Fig. 1(c).

The low-temperature XRD studies [7] and Raman data [5] suggested a further reduction of symmetry below $\approx 90 \mathrm{~K}$, 

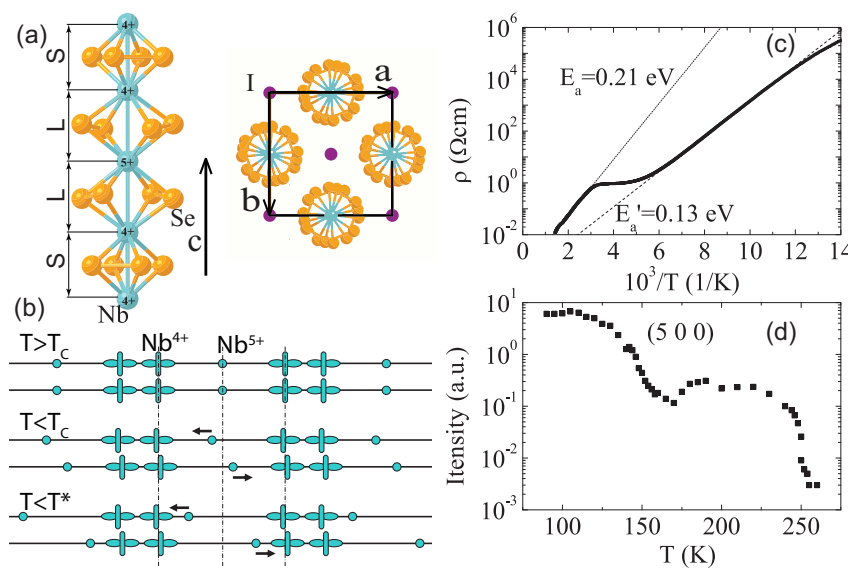

FIG. 1. (a) Schematic structure of an individual chain in $\left(\mathrm{NbSe}_{4}\right)_{3} \mathrm{I}$ (left), together with a view of the unit cell perpendicular to $c$ direction (right). (b) Schematic of $\mathrm{Nb}^{5+}$ displacements (not to scale) with $\mathrm{Se}_{4}^{4-}$ rectangles and $\mathrm{I}^{-}$ions not shown. (c) Temperature dependence of resistivity shows the two characteristic temperatures $T_{C}=274 \mathrm{~K}$ [the inflection point of the $\left.d(\log \rho) / d(1 / T)\right]$ and $T^{*} \approx$ $160 \mathrm{~K}$, with indicated activation energies $E_{a}$ for the high $\left(T>T_{C}\right)$ and the low $\left(T<T^{*}\right)$ temperature phases. (d) The $T$ dependence

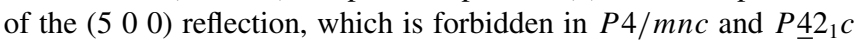
$[7,12]$, providing evidence for two successive phase transitions.

demonstrated through the appearance of the (003) reflection (forbidden in $D_{4 h}^{6}$ and $D_{2 d}^{4}$ symmetry phases) and additional phonon modes, respectively. At $30 \mathrm{~K}$ the $\mathrm{Nb}-\mathrm{Nb}$ spacings are 3.37-3.10-3.11-3.32-3.16-2.98 $\AA$ with $P \underline{4}\left(S_{4}^{1}\right)$ space group. Defining long and short distances as $L>3.31 \AA$ and $S<3.17 \AA$, respectively, an approximate $L S S L S S$ stacking is obtained, as presented in Fig. 1(b). Crystal remains AFE with opposite ionic displacements between the neighboring chains. The (500) Bragg reflection is allowed only in the $P \underline{4}$ space group. Our XRD data show that this reflection rises in intensity by two orders of magnitude below $T^{*}$, while between $T^{*}$ and $T_{C}$ the intensity is constant. We should note that from the available literature data, studies (including ours) have usually been done on the type-II compounds.

Space group analysis and Raman data in Ref. [5] showed that the symmetry of the order parameters below $T_{C}$ and $T^{*}$ are of $B_{1 u}$ and $A_{2}$ symmetries, respectively. Both $P \underline{4} 2_{1} c$ and $P 4$ space groups support AFM order between the chains.

Tight-binding calculations, considering $d_{z}^{2}$ orbitals of six in-chain $\mathrm{Nb}$ atoms, suggest that six bands are formed, two of which are fully occupied with four $\mathrm{Nb}^{4+} d_{z}^{2}$ electrons [8]. According to calculations the gap around the Fermi level is approximately $1 \mathrm{eV}$ (about a factor of 2 larger than the experimentally measured [9]). Considering just slightly less stable SSLSSL stacking (as the one observed below $T^{*}$ ), calculations have shown that the band gap narrows by factor of $\approx 1.5$, accompanied by a stronger $\mathrm{Nb}^{5+}$ displacements from equidistant positions than in the $L L S L L S$ phase.

Electron-phonon interactions have been initially investigated by studying the softening of Raman modes below $T_{C}$ $[5,10]$. The soft mode at $80 \mathrm{~cm}^{-1}$ was observed $[5,10]$ together with an additional mode at $30 \mathrm{~cm}^{-1}$ which disappeared below $100 \mathrm{~K}$ [5]. However, optical pump-probe [11] and neutron studies [12] revealed an additional soft mode at $12 \mathrm{~cm}^{-1}$. Based on the incomplete softening of the modes in Dvorsek et al. [11], authors have concluded that these modes are not the proper soft modes of the system. It was argued that the softening only reveals coupling between phonons and the electronic order parameter which undergoes an order-disorder transition at $T_{C}$. On the other hand, radio frequency dielectric response [4] reveals behavior characteristic for cooperative processes as in CDWs or similar collective states $[13,14]$, arguing in favor of correlated displacive ionic motion.

Here we present systematic temperature dependent femtosecond (fs) pump-probe studies combined with Raman spectroscopy to investigate the interplay between the structural and electronic degrees of freedom in $\left(\mathrm{NbSe}_{4}\right)_{3} \mathrm{I}$ single crystals, which were characterized by dc transport and XRD measurements. We demonstrate that the $\mathrm{pJT}$ structural transformation is gradual: first below $T_{C}$ it exhibits ferrodistortive order, while below $T^{*}=160 \mathrm{~K}$ an AFE order between chains sets in, as evidenced by a sudden enhancement of Raman modes and the onset of suppression of the dielectric constant [4].

\section{EXPERIMENT}

$\left(\mathrm{NbSe}_{4}\right)_{3} \mathrm{I}$ samples of typically $2 \times 2 \times 10 \mathrm{~mm}^{3}$ in size have been used. The samples were cleaved along the (110) plane. In the pump-probe experiments we studied $a^{\prime}(c c) a^{\prime}$ geometry, i.e., the probe beam was polarized along the chain [001] direction, while the pump beam was propagating along the $a^{\prime}$ [110] direction.

We used two different Ti:sapphire laser systems: (a) regenerative amplifier with $300 \mathrm{kHz}$ repetition rate and 60 fs pulse width and (b) Spectra-Physics Tsunami oscillator with $80 \mathrm{MHz}$ repetition rate and 80 fs pulse width, used for preliminary results. In (a) we used a fast-scan technique for sweeping the time delay between pump and probe pulses over $120 \mathrm{ps,}$ providing frequency resolution of $80 \mathrm{GHz}$ (energy resolution of $0.3 \mathrm{~cm}^{-1}$ ) and high dynamic range [15]. Temperature dependent measurements using a helium flow cryostat were performed at a constant photoexcitation density (fluence) of $100 \mu \mathrm{J} / \mathrm{cm}^{2}$ in (a) and $160 \mathrm{~nJ} / \mathrm{cm}^{2}$ in (b). Pump and probe beams have been focused on 100 and $70 \mu \mathrm{m}$ spots, respectively.

Raman experiments were performed with a $514.5 \mathrm{~nm}$ argon laser line, with intensity set to $10 \mathrm{~mW}$ and focused on a $50 \mu \mathrm{m}$ spot. The spectra were recorded by a Horiba Jobin Yvon T6400 spectrometer in the backscattering configuration. Sample was cooled down to $10 \mathrm{~K}$ in a closed cycle helium cryostat.

XRD measurements were made on the XMaS beamline (BM28) at the European Synchrotron Radiation Facility (ESRF) in Grenoble, France. The sample was mounted inside a small closed-cycle cryostat on a Huber goniometer. The experiment was performed in reflection geometry by a monochromatic beam of energy $E=8 \mathrm{keV}$ from a $\mathrm{Si}$ (111) monochromator, on the scattering surface (110) plane of $\approx 1 \times 3 \mathrm{~mm}^{2}$ area. The sample was aligned at $50 \mathrm{~K}$, where the Bragg peaks corresponding to the $P \underline{4}$ space group of the low-temperature structure were clearly observable [7]. The temperature evolution of the (500) peak, forbidden in the higher temperature phases, has been followed up to RT. 


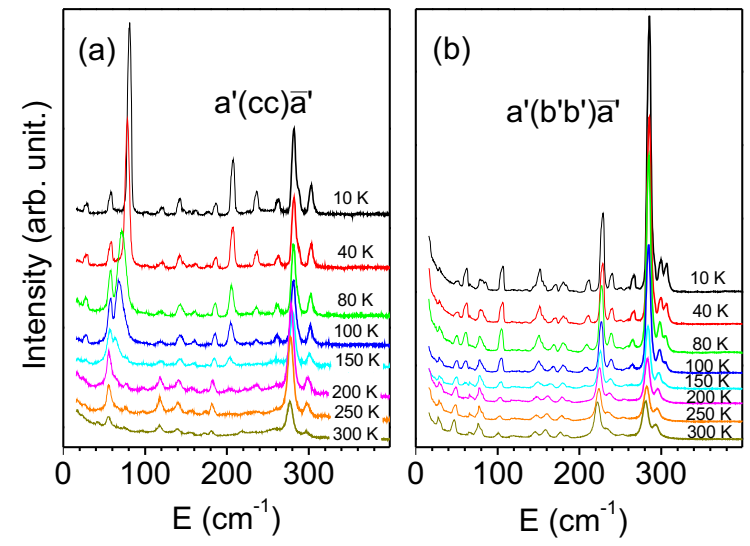

FIG. 2. Temperature dependence of Raman spectra with polarization (a) parallel and (b) perpendicular to chains.

\section{EXPERIMENTAL RESULTS}

\section{A. Temperature dependent Raman spectroscopy}

In Raman scattering measurement we used single crystals with (110) surfaces. Figure 2 shows the temperature dependence of the Raman (Stokes) spectra up to $400 \mathrm{~cm}^{-1}$ in the $a^{\prime}(c c) \bar{a}^{\prime}$ and $a^{\prime}\left(b^{\prime} b^{\prime}\right) \bar{a}^{\prime}$ polarization configuration, i.e., with light polarization along the $c$ (chain) and $b^{\prime} \|[1 \overline{1} 0]$ axis. The spectra show pronounced temperature dependence, especially in the $a^{\prime}(c c) \bar{a}^{\prime}$ configuration, Fig. 2(a), which is consistent with the published data [5]. Only a few phonon modes are not affected by the structural phase transitions. Such are, e.g., modes at 120 and $282 \mathrm{~cm}^{-1}$ in $a^{\prime}(c c) \bar{a}^{\prime}$ and $50 \mathrm{~cm}^{-1}$ in $a^{\prime}\left(b^{\prime} b^{\prime}\right) \bar{a}^{\prime}$ geometry. Their intensities follow the $I(T)=I_{0}[1+N(E, T)]$ dependence, where $N(E, T)$ is the Bose factor, while the weak temperature induced redshift of the peak position $E(T)$ and the increase of the linewidth $\operatorname{FWHM}(T)$ can be attributed to anharmonic effects [16,17].

We have fitted Raman modes in $a^{\prime}(c c) \bar{a}^{\prime}$ geometry using Gaussian, which gave a better fit than using Lorentzian. Fitting parameters for modes observed below $120 \mathrm{~cm}^{-1}$ are shown in Fig. 3. The linewidths are limited by the $\approx 4 \mathrm{~cm}^{-1}$ instrumental resolution.

Majority of the phonon modes display a weak softening on the order of a percent, yet they show a dramatic decrease in intensity upon increasing the temperature. Prime examples are the modes at 28, 207, 236, 262, and $288 \mathrm{~cm}^{-1}$ (side mode of strong mode at $\left.282 \mathrm{~cm}^{-1}\right)$ in $a^{\prime}(c c) \bar{a}^{\prime}$ and at 60, 104, 150 , and $285 \mathrm{~cm}^{-1}$ in $a^{\prime}\left(b^{\prime} b^{\prime}\right) \bar{a}^{\prime}$ geometry. The anomalous $T$ dependence of mode intensities can be exemplified on the $28 \mathrm{~cm}^{-1}$ mode in the $a^{\prime}(c c) \bar{a}^{\prime}$ configuration, where the Bose factor would give rise to an increase of the Stokes intensity by a factor of 8 upon increasing the temperature from 4 to $300 \mathrm{~K}$. Instead, the mode shows a dramatic loss in intensity at around $T^{*}$.

Finally, the $80 \mathrm{~cm}^{-1}$ mode in $a^{\prime}(c c) \bar{a}^{\prime}$ geometry shows pronounced softening $(\sim 15 \%-20 \%)$ in addition to a dramatic decrease in intensity upon increasing the temperature. The mode was argued to be the soft mode related to the phase transition at $T_{C}[5,10]$, and to display an anticrossing with the $60 \mathrm{~cm}^{-1}$ mode around $200 \mathrm{~K}$. Before addressing this question with time-resolved spectroscopy, we should point out that
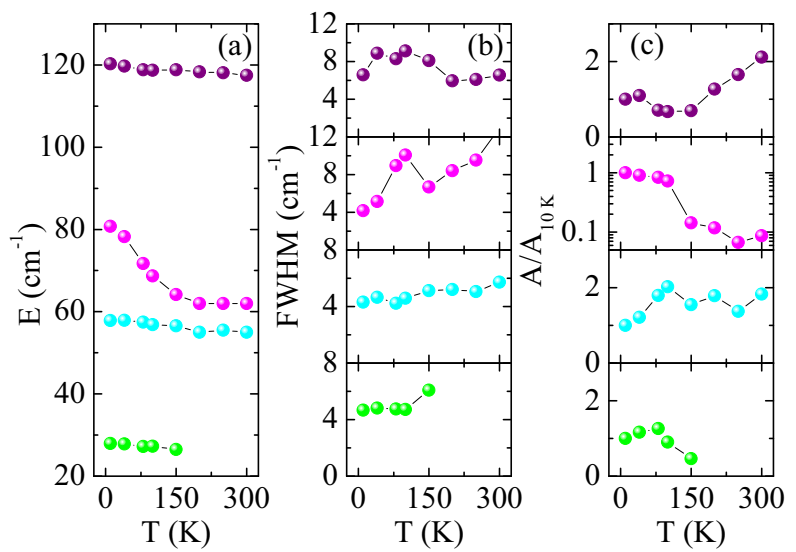

FIG. 3. Temperature dependence of $28,60,80$, and $120 \mathrm{~cm}^{-1}$ mode parameters obtained by fitting Raman data in $a^{\prime}(c c) \bar{a}^{\prime}$ geometry. (a)-(c) The $T$ dependence of phonon energies $E$, linewidths (FWHM), and integrated intensities normalized by $10 \mathrm{~K}$ values, $A / A_{10 \mathrm{~K}}$, respectively.

most of the phonon modes do not show major changes in the vicinity of $T_{C}$. In fact, they display much stronger changes close to $T^{*}$, displaying a dramatic increase in intensity upon cooling. Indeed, both transport and XRD data (Fig. 1) suggest the second phase transition at $T^{*}$ with further lowering of the symmetry.

\section{B. Temperature dependent study of transient reflectivity}

The femtosecond real-time spectroscopy has several advantages over the conventional Raman spectroscopy in the low energy range $[11,15,18-20]$. The energy resolution is limited by the scan length and not by the spectrometer or by the Rayleigh scattering. Indeed, the resolution of $0.1 \mathrm{~cm}^{-1}$ can be reached [15]. Moreover, the access to phase enables one to separate coherently driven modes of similar frequencies $[11,19]$. Furthermore, optical phonons down to $3 \mathrm{~cm}^{-1}$ can be accessed [18]. Finally, a dynamic range as high as $10^{4}$ has been demonstrated [15]. As such, the method is ideally suited to address some of the important issues related to phase transitions in $\left(\mathrm{NbSe}_{4}\right)_{3} \mathrm{I}$.

Figure 4(a) shows the photoinduced reflectivity transient recorded at $10 \mathrm{~K}$ with the probe polarization parallel to the $c$ axis. The photoinduced transient can be decomposed into several components. In addition to a decaying (overdamped) component(s) numerous oscillatory components due to coherently excited Raman active phonons can be observed. At higher temperatures the modes' dampings increase, as shown in Fig. 4(b), while their amplitudes show a nonmonotonic behavior, which is the most apparent for the mode with the period of $2.8 \mathrm{ps}\left(12 \mathrm{~cm}^{-1}\right)$. To obtain detailed information on their temperature dependence we performed full analysis of all the Fourier and decaying components.

We have developed an iterative algorithm that fits transient reflectivity data using three steps. In the first step we determine the overdamped components (typically electronic response), given by $A_{i} e^{-\Gamma_{i} t}\left(A_{i}\right.$ is the amplitude, $\Gamma_{i}$ is the damping) in the time domain and subtract them from the original transient. The best fit is obtained by two overdamped components and an 


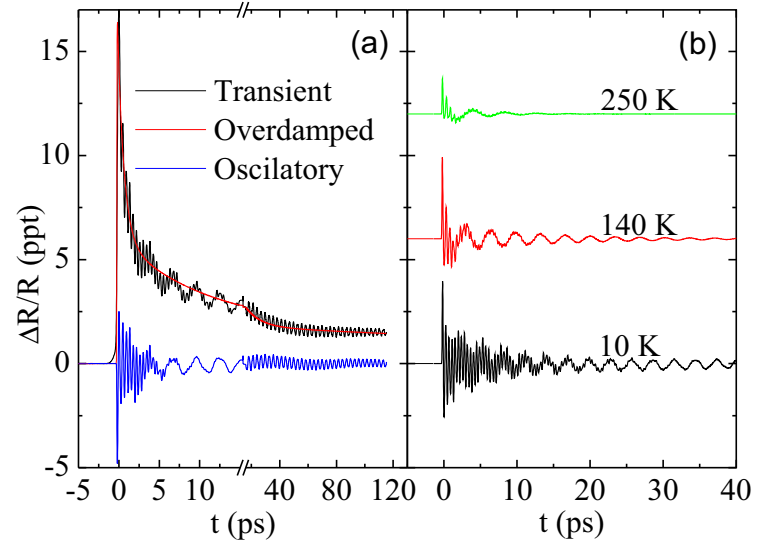

FIG. 4. (a) Photoinduced reflectivity transient recorded at $10 \mathrm{~K}$ (solid black line), decomposed into an overdamped (electronic) response which includes an offset at large time delays due to transient heating (red line) and an oscillatory response due to displacively driven coherent phonons (blue line). (b) Oscillatory components recorded at three selected temperatures.

offset (component with $\Gamma \rightarrow 0$ ), which can be attributed to a bolometric response (sample heating). The residual [Fig. 4(b)] is used in the second step to extract the initial parameters of the oscillatory components by fitting Lorentzians to the fast Fourier transform (FFT), Fig. 5(a). Here each oscillatory component is given by the expression $A_{i} e^{-\Gamma_{i} t} \cos \left(2 \pi E_{i} t+\right.$ $\phi_{i}$ ), where $A_{i}$ is the amplitude, $E_{i}$ is the frequency (later regarded as phonon energy), $\Gamma_{i}$ is the damping, and $\phi_{i}$ is the phase of the $i$ th mode. In the third step we use the initial parameters from the previous step and optimize them in the time domain, by fitting one component at a time (starting with the strongest mode) and subtracting all the previously fitted components. The entire procedure is performed in a few iterations, so that both the overdamped and the oscillatory components are extracted in a self-consistent way. For the fitting in the time domain all the components have been
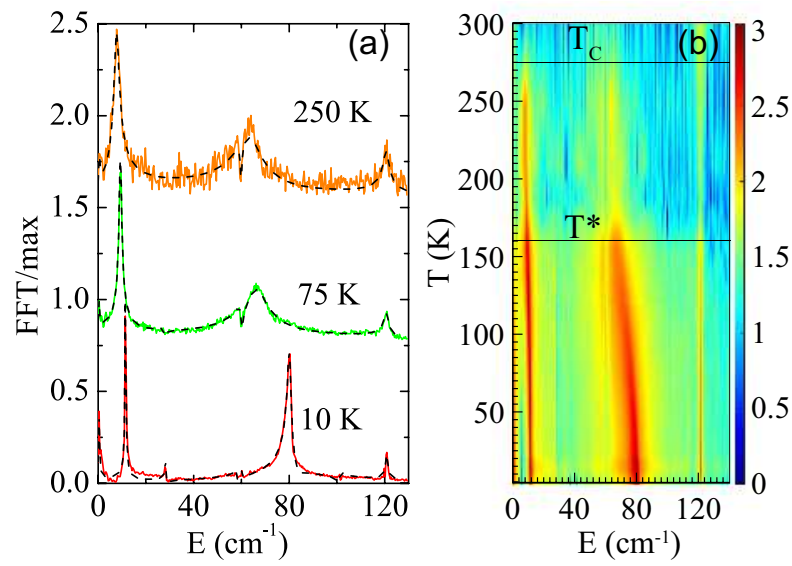

FIG. 5. (a) The FFT amplitude spectra of the oscillatory response [see Fig. 4(b)] at selected temperatures together with corresponding fits (dashed gray lines). The data have been normalized and shifted vertically for clarity. (b) The temperature evolution of the FFT spectra. Intensity, given by color coding, is on the logarithmic scale.

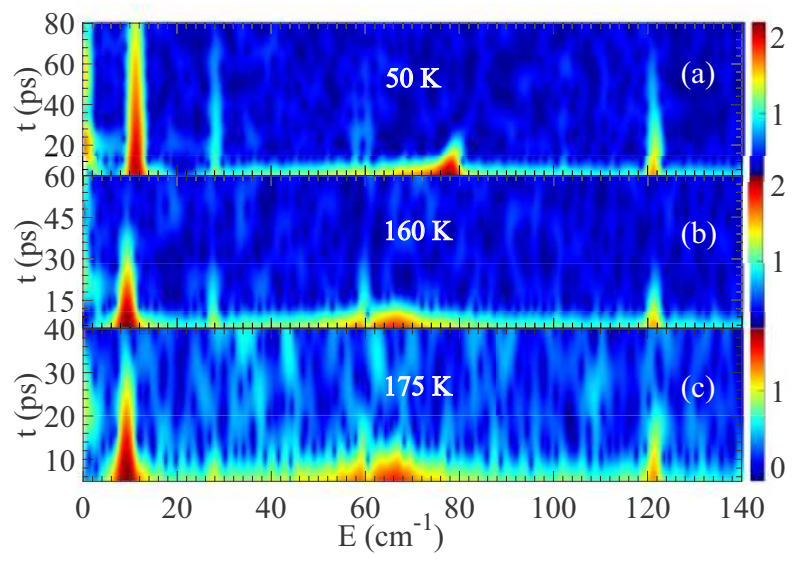

FIG. 6. Time evolution of the phonon spectra obtained by timewindowed FFT (with Gaussian window of $10 \mathrm{ps}$ ). The intensity scale is logarithmic. The data are recorded at $0.1 \mathrm{~mJ} / \mathrm{cm}^{2}$ at (a) $50 \mathrm{~K}$, (b) $160 \mathrm{~K}$, and (c) $175 \mathrm{~K}$. From (b) and (c) it follows that the 60 and $80 \mathrm{~cm}^{-1}$ modes are decoupled even close to phase transition.

analytically convoluted with the pulse intensity autocorrelation function. Note that amplitudes determined in the time domain $A_{i}$ correspond to an integrated intensities of the modes in the frequency domain.

The observed modes-see Fig. 5(b) - are generally consistent with Raman data in the same $a^{\prime}(c c) \bar{a}^{\prime}$ configuration. However, due to the lower low-energy cutoff with respect to Raman we observe an additional narrow mode at $12 \mathrm{~cm}^{-1}$ that displays comparable softening as the $80 \mathrm{~cm}^{-1}$ mode [11]. As some weak modes are barely resolved in the FFT spectra, in Fig. 6 we present the time-windowed FFT analysis. The $28 \mathrm{~cm}^{-1}$ mode is clearly observed even at $175 \mathrm{~K}$. Moreover, the mode at $60 \mathrm{~cm}^{-1}$ turns out to be a doublet, with the modes separated by only $3 \mathrm{~cm}^{-1}$, Fig. 6(a). They can be clearly resolved at least up to $100 \mathrm{~K}$, where the lower energy mode seems to disappear. Finally, we observe a mode at $0.5 \mathrm{~cm}^{-1}(17 \mathrm{GHz})$. We attribute this mode to the pump-induced shockwave propagating into the bulk [20,21]. Although we included this mode during the fitting, we will not consider it further.

Figure 5(b) presents the temperature dependence of the low energy phonon spectra. In addition to a clear softening of 12 and $80 \mathrm{~cm}^{-1}$ modes we note a strong dependence of FFT peaks on temperature, displaying the same trend as the Raman data. Indeed, clear intensity drop around $160 \mathrm{~K}$ is suggestive of a phase transition at $T^{*}$. Above $100 \mathrm{~K}$ the modes at 80 and $60 \mathrm{~cm}^{-1}$ start to spectrally overlap. However, it is clear from the data in Fig. 5 that the modes do not interact. Indeed, our data are at odds with the proposed phonon anticrossing scenario, suggesting a complete softening of the $60 \mathrm{~cm}^{-1}$ mode at $T_{C}$ [5].

The weak mode at $28 \mathrm{~cm}^{-1}$ was observed to vanish near $100 \mathrm{~K}$ in the early Raman study [5]. It was attributed to the soft mode of the low-temperature phase transition. Our data demonstrate that the mode is indeed observed only up to $T^{*}$, but showing very weak softening $(\sim 1 \%)$, Figs. 5 and 6 . Thus it is unlikely to be the soft mode of the low-temperature phase transition; more likely it experiences similar loss of intensity 

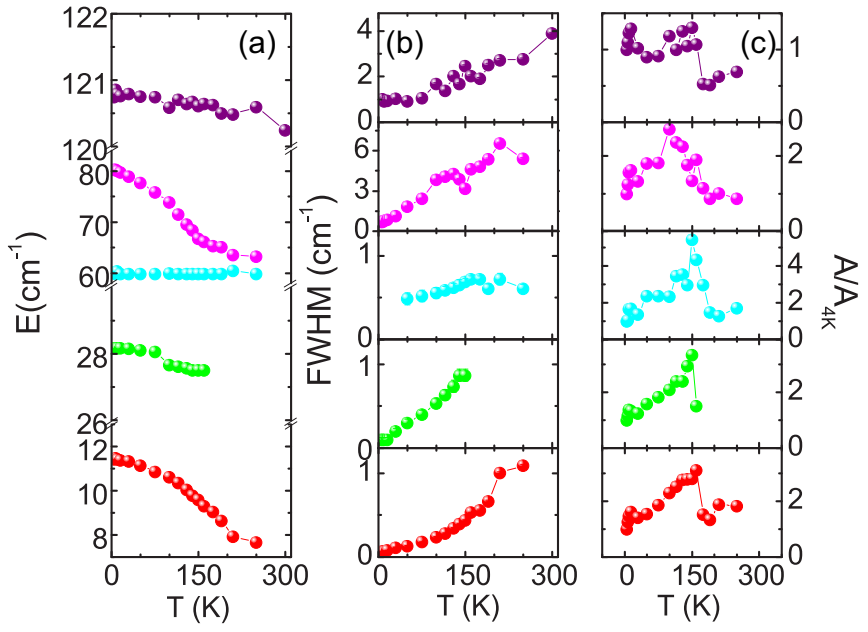

FIG. 7. Temperature dependence of mode parameters obtained by fitting the time domain data. (a)-(c) The $T$ dependence of phonon energies $E$, linewidth (FWHM of the power spectra), and normalized mode amplitudes (oscillator strengths), respectively.

above $T^{*}$ as most of the low energy modes, with its intensity falling below the resolution limit above $T^{*}$. Similarly to some other weak modes that are barely resolved in the FFT spectra of the entire transient, the $28 \mathrm{~cm}^{-1}$ mode can be clearly seen by looking at the time-windowed FFT, shown in Fig. 6. Here Gaussian windowing with $10 \mathrm{ps}$ full width half maximum was used, aiming at higher energy resolution.

Figure 7 presents the extracted temperature dependence of (a) phonon energies, (b) linewidths (FWHM), and (c) their oscillator strengths. We chose to plot FWHM values of power spectra (square of the spectra in Figs. 5 and 6) instead of the relaxation rate $\Gamma$, so that we can directly compare the Raman and the time-domain results. Note the relation between the two: $\mathrm{FWHM}=\Gamma / 2 \pi$. Due to its low intensity, in this analysis we have omitted the mode at $57 \mathrm{~cm}^{-1}$ (lower part of the doublet at $60 \mathrm{~cm}^{-1}$ ). Of all the modes, only the $121 \mathrm{~cm}^{-1}$ mode is clearly resolved above $T_{C}$ [see also Fig. 5(b)]. We can see that three modes $\left(12,28\right.$, and $\left.80 \mathrm{~cm}^{-1}\right)$ are much more affected by the temperature than the modes at 60 and $121 \mathrm{~cm}^{-1}$, whose weak softening and weakly $T$-dependent damping $\left(\mathrm{FWHM}_{300 \mathrm{~K}} / \mathrm{FWHM}_{4 \mathrm{~K}} \sim 2\right)$ can be attributed to anharmonic effects. The pronounced softening, observed for the 12 and $80 \mathrm{~cm}^{-1}$ modes, and large changes of damping with temperature $\left(\mathrm{FWHM}_{300 \mathrm{~K}} / \mathrm{FWHM}_{4 \mathrm{~K}} \sim 10\right)$ for all three modes, imply strong electron-phonon coupling. On the other hand, 12 and $80 \mathrm{~cm}^{-1}$ modes exhibit peculiar s-shape $T$ dependence of phonon energy. Such a trend is not expected for a soft mode [15].

The temperature dependence of amplitudes (oscillator strengths) is also nontrivial. In general, the change in reflectivity due to the coherently driven phonon depends on two Raman-like tensors [22] which account for the phononinduced modulation of the susceptibility and for the driving force, respectively [19,22,23]. Both tensors change upon changing the symmetry. This way breaking of the inversion symmetry can raise Raman activity of previously IR active mode. This means that in the low-temperature phase these modes can be both excited and probed by the coherent phonon

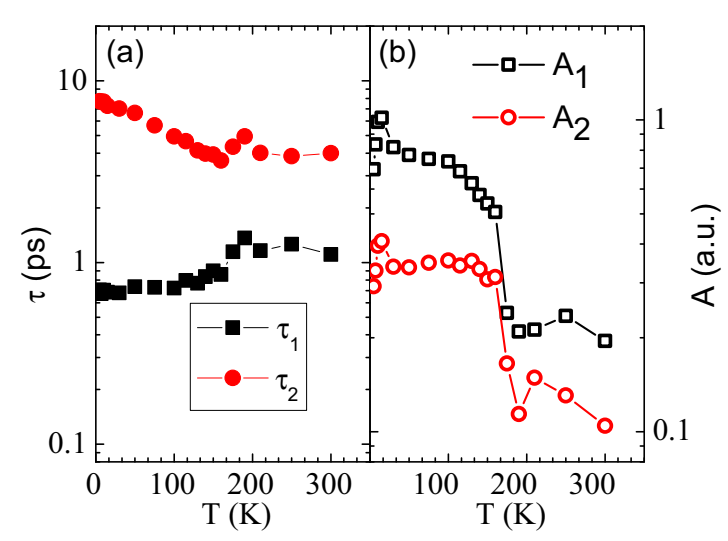

FIG. 8. The temperature dependence of (a) relaxation times and (b) amplitudes of the two relaxation modes.

spectroscopy. Indeed, the pronounced drop in amplitudes near $T^{*}$, which is similar to Raman data, suggests that upon cooling through $T^{*}$ further symmetry reduction takes place.

In addition to the anomaly at $T^{*}$ we should mention also the decreasing amplitude upon further cooling, which is particularly pronounced for 12 and $28 \mathrm{~cm}^{-1}$ modes. This behavior is opposite to the one in charge density wave systems $[15,19]$, where the amplitudes are nearly constant below the CDW transition temperature [19]. As elaborated in Sec. IV, the difference could be attributed to a different excitation/probing mechanism in $\left(\mathrm{NbSe}_{4}\right)_{3} \mathrm{I}$ as opposed to standard CDW systems.

Finally, Fig. 8 presents the analysis of the incoherent (overdamped) part of the reflectivity transient. The data were fitted by a sum of two exponentially decaying components, with strongly $T$-dependent amplitudes and decay times. While the amplitudes show a similar increase upon cooling through $T^{*}$ as the oscillator strengths of the phonon modes, they increase further upon cooling down to $4 \mathrm{~K}$. The relaxation times also show a pronounced temperature dependence with anomalies near $T^{*}$. In fact, the temperature dependence of both the time scales and the amplitudes is similar as in CDW systems, where the overdamped components were associated with the collective response of the electronic order parameter $[15,19,24]$. The nearly identical trend observed here, supported by the strong renormalization of several modes at $T^{*}$, suggests such collective mode description may be appropriate in the $\left(\mathrm{NbSe}_{4}\right)_{3} \mathrm{I}$ as well.

\section{DISCUSSION AND CONCLUSIONS}

The combined XRD, Raman, and optical time-resolved spectroscopy data suggest that $\left(\mathrm{NbSe}_{4}\right)_{3} \mathrm{I}$ undergoes two phase transitions upon cooling down from room temperature, at $T_{C}=274 \mathrm{~K}$ and at $T^{*} \approx 160 \mathrm{~K}$. Several Raman silent, long wavelength phonons become visible in Raman below $T_{C}$, which is consistent with the group theoretical analysis $[5,25]$. Comparison with the published IR data $[9,25]$ reveals that at least the low-temperature Raman active modes at 60,80 , and $200 \mathrm{~cm}^{-1}$ could originate from the modes that are (only) IR active in the high temperature phase. Such a scenario is likely responsible also for the appearance of 12 and $28 \mathrm{~cm}^{-1}$ modes 
at low temperatures (the first does not seem to be IR active either $[9,25])$.

The fingerprint of the transition at $T^{*}$ is observed in the Raman and pump-probe response as a sudden increase of Raman intensities and coherent phonon amplitudes, respectively. Below $T^{*}$ the coherent phonon amplitudes start to decrease again, accompanied by the increase of the phonon energies and the reduction of dampings. Furthermore, at $T^{*}$ relaxation times of the overdamped components split apart and their amplitudes increase, as shown in Fig. 8.

Comparison of the phonon modes' parameters extracted from Raman (Fig. 3) and time-resolved (Fig. 7) data, shows a good agreement in modes energies and dampings. As far as the linewidths are concerned, Raman data are largely limited by the instrumental resolution of $\approx 4 \mathrm{~cm}^{-1}$. Moreover, in both experiments amplitudes of several modes display a sudden jump in intensity upon cooling through $T^{*}$, consistent with lowering of the symmetry. Their $T$ dependencies, however, show pronounced differences, related to the nature of their excitation, as elaborated below.

As mentioned above, existence of the second structural phase transition in $\left(\mathrm{NbSe}_{4}\right)_{3} \mathrm{I}$ was indicated already in earlier XRD and Raman studies [2,5] at $\approx 100 \mathrm{~K}$. However, in our samples, coming from another batch, all the methods used (pump-probe, Raman, XRD and dielectric spectroscopy [4]) consistently point to one transition temperature of $T^{*} \approx 160 \mathrm{~K}$. The accompanying transport data in Ref. [5] display a plateau in resistivity near $T_{C}$, which is characteristic for type-II samples, as the ones studied in this work. On the other hand, this plateau is not expressed as much as in our samples, Fig. 1(c). Thus it is possible that the second phase transition is sensitive to the sample purity, unlike the high temperature phase transition, which is consistently observed at $T_{C} \approx 274 \mathrm{~K}$.

Ferrodistortive in-chain displacements appear below $T_{C}$, changing niobium in-chain distances from $L L S L L S$ to LISLIS stacking, accompanied by the shifting of chains against each other in the $c$ direction [3]. Below $T^{*}$ niobium in-chain distances further change to approximate LSSLSS stacking [7]. The band calculations in Ref. [8] suggest two stable distortions of $\mathrm{MX}_{4}$ chain, corresponding to $L L S L L S$ and $L S S L S S$ stacking of $\mathrm{Nb}$ atoms, with the band gap of the former being about $3 / 2$ of the band gap in the latter. The activation behavior of dc conductivity in Fig. 1(c) (see also Ref. [1]) gives about the same ratio of activation energies above RT and below $T^{*}$. Thus the LISLIS stacking in the temperature region between $T_{C}$ and $T^{*}$ could be considered as an intermediate between stable $L L S L L S$ and $L S S L S S \mathrm{Nb}$ stackings.

The evolution of the in-chain stacking of $\mathrm{Nb}^{5+}$ ions can be considered similarly as in the model proposed in Ref. [4]. The instability of $L L S L L S$ stacking towards $L S S L S S$ below $T_{C}$ is represented by the formation of a double well potential around the site of an isolated $\mathrm{Nb}^{5+}$ ion. By forming such a potential, the loss of inversion symmetry permits both the FE and AFE in-chain ordering. The degeneracy between the possible FE and AFE orders is likely broken by the opposite shifts of the neighboring chains and interaction with $\mathrm{Se}_{4}^{4-}$ rectangles, raising an in-chain asymmetry of the potential which reverses direction between chains. Such asymmetry is favoring FE inchain and the AFE interchain ordering. At higher temperatures $\mathrm{Nb}^{5+}$ is fluctuating between the two wells, with its average position closer to the deeper well. Thus, LISLIS stacking with an in-chain FE order and a weak interchain AFE order forms below $T_{C}$. Further decrease of temperature below $T^{*}$ reduces the fluctuations and establishes the long range AFE order and concomitant LSSLSS stacking. In the presence of the fluctuations, the material is highly polarizable and exhibits colossal dielectric constant, i.e., effective potential curvature for $\mathrm{Nb}^{5+}$ is fairly small. The same thermal fluctuations tend to destroy coherence of the order, so that the dielectric constant decreases when increasing the temperature towards $T_{C}$. On the other hand, upon decreasing the temperature below $T^{*}$ the long range AFE state forms and the dielectric constant again starts to decrease. From the value of $T^{*}$, it can be expected that the barrier height is just a few tens of meV. From the symmetry point of view, AFM interchain order is supported below $T_{C}$ in both $P \underline{4} 2_{1} C$ and $P \underline{4}$ phases.

By monitoring the temperature dependencies of the (005) reflection intensity (forbidden in the $P 4 / m n c$ and $P \underline{4} 2_{1} c$ symmetry phases, but allowed in the low-temperature $P \underline{4}$ phase), as well as of the phonon intensities measured by Raman and pump-probe spectroscopy, we infer that the structural displacements are much stronger below $T^{*}$ than for $T^{*}<$ $T<T_{C}$. Within the proposed scenario, where the appearance of Raman active modes below $T_{C}$ is governed by breaking of inversion symmetry, the temperature dependence of Raman intensities is expected to follow the square of the order parameter [i.e., the intensity of the (005) reflection], multiplied by $1+N(\omega, T)$. Indeed, the temperature dependence of the intensity of $80 \mathrm{~cm}^{-1}$ mode qualitatively follows this scenario, see Fig. 3. The same behavior is also seen for 207, 236, 262, and $288 \mathrm{~cm}^{-1}$ modes, despite the fact that these modes display only negligible softening. The temperature dependence of the coherently driven phonons, shown in Fig. 7(c), displays a jump at the $T^{*}$, consistent with the inversion symmetry breaking scenario. However, the amplitudes decrease upon cooling to liquid He temperature.

Other members of the $\left(\mathrm{MSe}_{4}\right)_{n} \mathrm{I}$ compound family examined so far commonly undergo Peierls transition to a CDW ground state. The similarities between $\left(\mathrm{NbSe}_{4}\right)_{3} \mathrm{I}$ and CDWs, such as, e.g., colossal dielectric response [4], phasonlike frequency response $[4,13,14]$, and phonon softening [5], have already been pointed out in Ref. [4]. While our results do not support the proposed soft-mode behavior [5], we should note the nearly one-to-one correspondence between the temperature dependencies of overdamped components in $\left(\mathrm{NbSe}_{4}\right)_{3} \mathrm{I}$ and in the prototype quasi-1D CDW system $\mathrm{K}_{0.3} \mathrm{MoO}_{3}$ [15,19]. Indeed, their temperature dependence is consistent with the collective electronic mode, suggesting strong coupling between the electronic system and the lattice, as should be the case within the pJT scenario. Since 80 and $12 \mathrm{~cm}^{-1}$ modes are strongly affected by both structural transitions (the one at $T^{*}$ in particular), it is likely that these modes are predominantly given by the displacements of $\mathrm{Nb}$ ions. In view of this, the temperature dependence of the coherent phonon amplitudes below $T^{*}$ [Fig. 7(c)] could be viewed within the model presented in Ref. [19], where phonons are linearly coupled to the electronic order parameter [as opposed to CDWs, the coupling in the case of $\left(\mathrm{NbSe}_{4}\right)_{3} \mathrm{I}$ takes place at $q=0$ ]. As demonstrated in Fig. 5 of Ref. [19] the temperature dependence of mode amplitudes 
depends on excitation and probing mechanisms, which should depend on the probe photon energy. Qualitatively, the observed increase in amplitudes upon increasing the temperature within the low-temperature phase [Fig. 7(c)] is consistent with the scenario where both the excitation and probing proceed via the electronic part of order parameter [19]. Detailed simulations, considering different coupling strengths of different modes and the proper temperature dependence of the order parameter, are beyond the scope of this paper.

To summarize, we report on systematic temperature dependent Raman and femtosecond time-resolved study of lattice dynamics in $\left(\mathrm{NbSe}_{4}\right)_{3} \mathrm{I}$, combined with the dc transport and XRD measurements. We have shown that type-II $\left(\mathrm{NbSe}_{4}\right)_{3} \mathrm{I}$ undergoes two second-order structural phase transitions upon cooling from the room temperature. In addition to the well documented ferrodistortive phase transition of the pJT type at $T_{C}=$ $274 \mathrm{~K}$, we demonstrate a further reduction of symmetry, taking place at $T^{*} \approx 160 \mathrm{~K}$. The latter is AFE order to disorder transition, with a weak AFE order parameter still present up to $T_{C}$.
AFE order is probably related to interchain coupling and sensitive to sample purity. The collective character of the response and the peculiar temperature dependence of both the electronic relaxation and of the coherently driven phonons suggest a strong coupling between the electronic degrees of freedom and structure consistent with the pseudo-Jahn-Teller scenario.

\section{ACKNOWLEDGMENTS}

We would like to thank G. Baranović for the technical support and L. Degiorgi for providing us with the FTIR data. We want to acknowledge the technical support of the BM28 beamline staff members in the realization of XRD experiment. This study was funded from the FemtoBias project, the Grant Agreement 55 of the NEWFELPRO fellowship project (Grant Agreement No. 291823) cofinanced by the EU 7th Framework Programme 2007-2013 (MSCA-FP7-PEOPLE2011-COFUND).
[1] P. Gressier, A. Meerschaut, L. Guemas, and J. Rouxel, J. Solid State Chem. 51, 141 (1984).

[2] M. Izumi, T. Iwazumi, K. Uchinokura, R. Yoshizaki, and E. Matsuura, Solid State Commun. 51, 191 (1984).

[3] P. Gressier, L. Guemas, and A. Meerschaut, Mater. Res. Bull. 20, 539 (1985).

[4] D. Starešinić, P. Lunkenheimer, J. Hemberger, K. Biljaković, and A. Loidl, Phys. Rev. Lett. 96, 046402 (2006).

[5] T. Sekine and M. Izumi, Phys. Rev. B 38, 2012 (1988).

[6] I. B. Bersuker, Chem. Rev. 113, 1351 (2013).

[7] M. Izumi, T. Iwazumi, T. Seino, T. Sekine, and E. Matsuura, Synth. Met. 19, 863 (1987).

[8] P. Gressier, M.-H. Whangbo, A. Meerchaut, and J. Rouxel, Inorg. Chem. 23, 1221 (1984).

[9] V. Vescoli, F. Zwick, J. Voit, H. Berger, M. Zacchigna, L. Degiorgi, M. Grioni, and G. Grüner, Phys. Rev. Lett. 84, 1272 (2000).

[10] T. Sekine, K. Uchinokura, M. Izumi, and E. Matsuura, Solid State Commun. 52, 379 (1984).

[11] D. Dvorsek, V. V. Kabanov, K. Biljakovic, and D. Mihailovic, Phys. Rev. B 74, 085211 (2006).

[12] P. Monceau, L. Bernard, R. Currat, and F. Levy, Phys. B 156 \& 157, 20 (1989).

[13] P. B. Littlewood, Phys. Rev. B 36, 3108 (1987).
[14] D. Starešinić, K. Biljaković, W. Brütting, K. Hosseini, P. Monceau, H. Berger, and F. Levy, Phys. Rev. B. 65, 165109 (2002).

[15] H. Schäfer, V. V. Kabanov, M. Beyer, K. Biljakovic, and J. Demsar, Phys. Rev. Lett. 105, 066402 (2010).

[16] P. G. Klemens, Phys. Rev. 148, 845 (1966).

[17] J. Menendez and M. Cardona, Phys. Rev. B 29, 2051 (1984).

[18] H. Schaefer, M. Körber, A. Tomeljak, K. Biljakovic, H. Berger, and J. Demsar, Eur. Phys. J. 222, 1005 (2013).

[19] H. Schaefer, V. V. Kabanov, and J. Demsar, Phys. Rev. B 89, 045106 (2014).

[20] C. Thomsen, J. Strait, Z. Vardeny, H. J. Maris, J. Tauc, and J. J. Hauser, Phys. Rev. Lett. 53, 989 (1984).

[21] I. Bozovic, M. Schneider, Y. Xu, R. Sobolewski, Y. H. Ren, G. Lüpke, J. Demsar, A. J. Taylor, and M. Onellion, Phys. Rev. B 69, 132503 (2004).

[22] K. W. Kim, A. Pashkin, H. Schäfer, M. Beyer, M. Porer, T. Wolf, C. Bernhard, J. Demsar, R. Huber, and A. Leitenstorfer, Nat. Mater. 11, 497 (2012).

[23] T. E. Stevens, J. Kuhl, and R. Merlin, Phys. Rev. B 65, 144304 (2002).

[24] A. Tomeljak, H. Schäfer, D. Städter, M. Beyer, K. Biljakovic, and J. Demsar, Phys. Rev. Lett. 102, 066404 (2009).

[25] V. Zelezny, J. Petzelt, B. P. Gorshunov, A. A. Volkov, G. V. Kozlov, P. Monceau, and F. Levy, J. Phys.: Condens. Matter 1, 10585 (1989). 